\title{
Mechanical and microstructural integrity of nickel-titanium and stainless steel laser joined wires
}

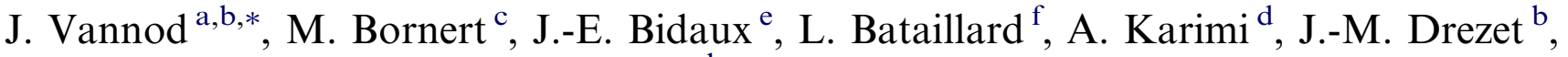 \\ M. Rappaz ${ }^{\mathrm{b}}$, A. Hessler-Wyser ${ }^{\mathrm{a}}$ \\ ${ }^{a}$ Centre Interdisciplinaire de Microscopie Electronique, Ecole Polytechnique Fédérale de Lausanne (EPFL), CH-1015 Lausanne, Switzerland \\ ${ }^{\mathrm{b}}$ Laboratoire de Simulation des Matériaux, EPFL, CH-1015 Lausanne, Switzerland \\ ${ }^{\mathrm{c}}$ Laboratoire Navier, Université Paris Est, Ecole des Ponts ParisTech, F-77455 Marne-la-Vallée, France \\ d Institut de Physique de la Matiére Condensée, EPFL, CH-1015 Lausanne, Switzerland \\ ${ }^{\mathrm{e}}$ University of Applied Sciences Western Switzerland, CH-1950 Sion, Switzerland \\ ${ }^{\mathrm{f}}$ Heraeus Medical Components Division, CH-1400 Yverdon-les-bains, Switzerland
}

Received 16 February 2011; received in revised form 14 June 2011; accepted 18 June 2011

Available online 16 August 2011

\begin{abstract}
The biomedical industry shows increasing interest in the joining of dissimilar metals, especially with the aim of developing devices that combine different mechanical and corrosive properties. As an example, nickel-titanium shape memory alloys joined to stainless steel are very promising for new invasive surgery devices, such as guidewires. A fracture mechanics study of such joined wires was carried out using in situ tensile testing and scanning electron microscopy imaging combined with chemical analysis, and revealed an unusual fracture behaviour at superelastic stress. Nanoindentation was performed to determine the mechanical properties of the welded area, which were used as an input for mechanical computation in order to understand this unexpected behaviour. Automated image correlation allowed verification of the mechanical modelling and a reduced stress-strain model is proposed to explain the special fracture mechanism. This study reveals the fact that tremendous property changes at the interface between the NiTi base wire and the weld area have more impact on the ultimate tensile strength than the chemical composition variation across the welded area.
\end{abstract}

(C) 2011 Acta Materialia Inc. Published by Elsevier Ltd. All rights reserved.

Keywords: Shape memory alloys (SMA); Laser welding; Tension testing; Stainless steel; Fracture

\section{Introduction}

Among the various materials that are used in biomedical engineering, nickel-titanium (NiTi) alloys play a major role due to their pseudoelastic, shape memory, corrosion and biocompatibility properties [1-3]. They are mainly used in the design of medical devices for invasive surgery, such as catheter guidewires, stents or coil anchors. In order to

\footnotetext{
* Corresponding author at: EPFL-SB-CIME-GE, Station 12, CH-1015 Lausanne, Switzerland. Tel.: +41 21693 6813; fax: +41 216934401.

E-mail addresses: jonas.vannod@a3.epfl.ch (J. Vannod), michel. rappaz@epfl.ch (M. Rappaz), aicha.hessler@epfl.ch (A. Hessler-Wyser).
}

extend their range of applications, there is strong interest in being able to join NiTi alloys with other biocompatible alloys, such as stainless steel (SS) or platinum alloys. The present study focuses on joining NiTi to SS submillimetric wires.

When joining dissimilar materials, a good starting point is to consider the multicomponent phase diagram of the various elements present in the base materials. Neglecting the presence of chromium in SS, since it has little impact on the weld composition and compound formation, the three main elements to be considered in this case are iron, nickel and titanium. Fig. 1 shows an isothermal cut of the $\mathrm{Fe}-\mathrm{Ni}-\mathrm{Ti}$ ternary phase diagram, according to Cacciamani 


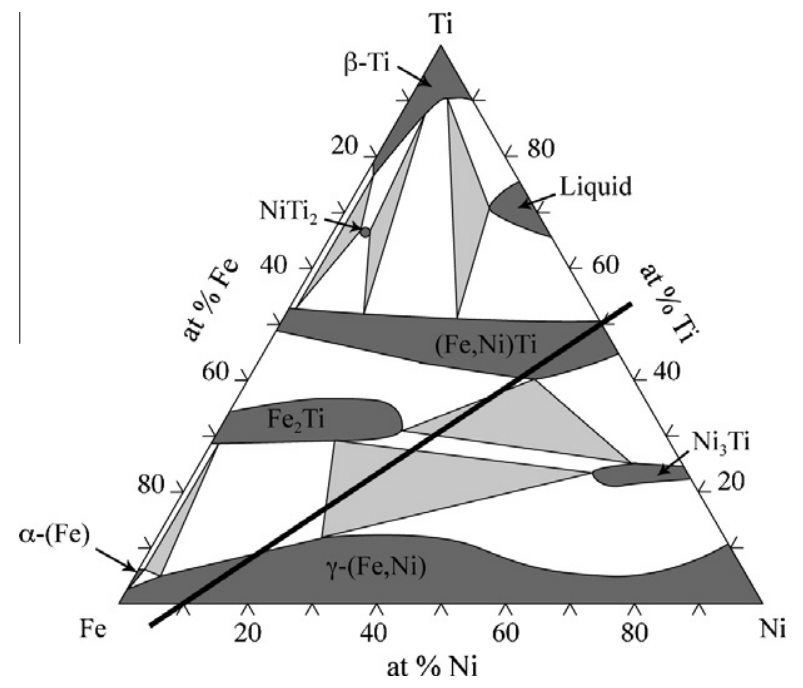

Fig. 1. Fe-Ni-Ti ternary phase diagram, isothermal cut at $1000^{\circ} \mathrm{C}[4]$.

et al. [4]. The black line connects the two initial phases of the base wires, i.e. SS in the lower left corner of the Gibbs simplex and NiTi in the middle of the right boundary.

Following the diffusion path theory described in 1963 by Kirkaldy [5], the joint can potentially exhibit all the phases appearing in single- or multi-phase regions of the phase diagram crossed by the diffusion path connecting the two nominal compositions. In the present case, $(\mathrm{Fe}, \mathrm{Ni})-\mathrm{Ti}$, $\mathrm{Fe}_{2} \mathrm{Ti}$ and $\mathrm{Ni}_{3} \mathrm{Ti}$ can form during welding of $\mathrm{NiTi}$ and SS, these intermetallic phases being known to be highly brittle $[6,7]$. It ishould be noted that the formation of intermetallic brittle phases is a common issue for most dissimilar laser welding systems in which steel is present, such as $\mathrm{Au}-\mathrm{SS}$ [8] and Al-SS [9]. The use of a kinetics-driven welding process, such as the laser welding used in the present study, may be a solution to inhibit the nucleation of these undesired compounds, as shown by Borrisutthekul et al. [10]. Indeed, laser welding is a very versatile process, which allows flexible control of the solidification parameters (cooling rate, thermal gradient, velocity) by an adjustment of the energy density and distribution. For example, increasing the power density and travel speed of the laser leads to a shallower molten pool, shorter interaction time and more rapid solidification conditions. At the same time, the phase fractions in the weld can be adapted via the socalled dilution factor of the two materials, by the careful positioning of the laser beam with respect to the interface separating the two base materials to be welded [11].

The conditions under which sound NiTi-SS laser welds can be produced are the topic of another contribution [12]. The main goal of this article is to characterize and better understand the deformation and fracture mechanisms that occur under tensile loading in sound NiTi-SS laser-welded wires. For this purpose, scanning electron microscopy (SEM) imaging will be used to observe the evolution of the welded wires during in situ tensile testing. Chemical analysis and nanoindentation measurements are then performed in the longitudinal section of welded wires in order to deduce the effective elastic modulus and yield strength of the various zones. These data are then used in deformation computations, which give access to the inhomogeneous stress-strain field in the wires. The computed strain field is finally compared with the actual deformation field of the wires measured by digital image correlation. As a conclusion, the relative contributions of the superelasticity of NiTi and the brittleness of the intermetallic phases are discussed.

\section{Experimental procedure}

The samples for the present investigations were all produced by welding together wires of NiTi $(50.8 \mathrm{wt} . \% \mathrm{Ni})$ and SS (grade 304L), $300 \mu \mathrm{m}$ in diameter. Prior to welding, the NiTi wires were chemically etched in a hydrofluoric and nitric acid aqueous solution to remove the titanium black oxide layer formed during their production prior to welding. The SS wires were used as received (wire-drawn without annealing). Welds were made with a Nd:YAG pulsed laser fixed on a positioning device that allowed careful alignment of the two wires with respect to the laser beam. The pulse energy and duration were adjusted to produce a complete transverse weld (see Fig. 2). The time between each pulse was set to be long enough to allow solidification and heat diffusion over the wire radius, and thus to obtain isotherms almost normal to the wire axis, right at the beginning of the next pulse. A laminar flux of pure argon was blown during welding in order to avoid titanium oxidation. Some welded couples were mechanically ground to reduce their initial diameter from $300 \mu \mathrm{m}$ to $200 \mu \mathrm{m}$ (see Fig. 2, dashed lines) in order to remove surface defects and inhomogeneities, and to ensure a constant diameter along the welded couple.

Longitudinal sections of the welded specimens, such as the one shown in Fig. 2, were observed by SEM using backscattered electrons (BSE, atomic number contrast)

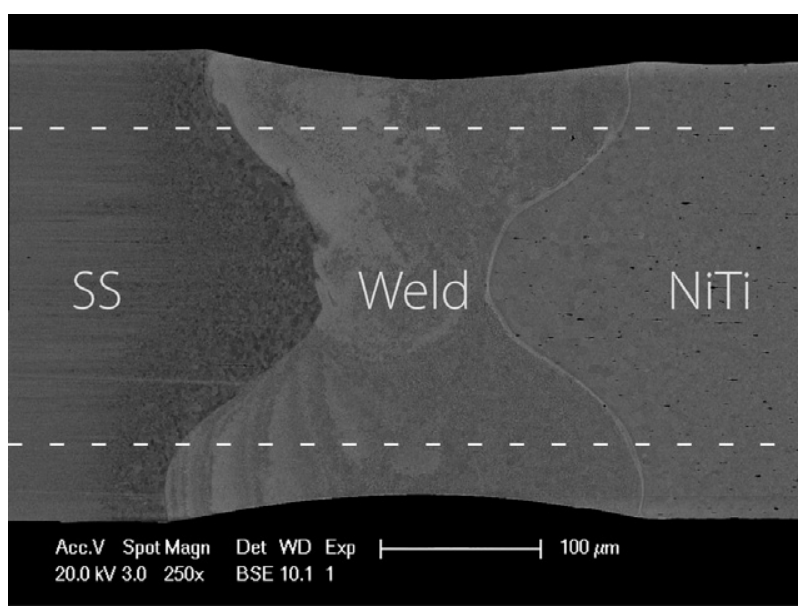

Fig. 2. SEM BSE micrograph showing a longitudinal cut of a welded NiTi-SS couple. The initial diameter is $300 \mu \mathrm{m}$; dashed lines indicate the $200 \mu \mathrm{m}$ reduced sample diameter. 
after mechanical polishing with $\mathrm{SiC}$ grinding disks and aqueous silica solution to obtain mirror-polished sections.

\subsection{In situ tensile testing}

In situ mechanical testing was conducted in an FEIXLF 30 scanning electron microscope, using a Deben ${ }^{\circ}$ tensile device with a capstan grip that ensured the correct positioning of and minimal damage to the wires. The roughness of the specimen surface after mechanical grinding from $300 \mu \mathrm{m}$ to $200 \mu \mathrm{m}$ was used for automatic processing by means of digital image correlation (DIC) techniques in order to deduce the inhomogeneous deformation field (see Ref. 2.4). In this case, the welded region, which was no longer apparent, was roughly positioned (typically $1 \mathrm{~mm}$ accuracy) in the middle of the $5 \mathrm{~cm}$ grip gap. The zone of interest was located by energy-dispersive X-ray (EDX) spectroscopy.

SEM secondary electron (SE) images during in situ tensile testing were made with a high definition (around 4000 pixels in width). As the tensile device has some compliance, the specimen might move slightly during the arrest needed to record the picture. For this reason, rather than using a fast scanning beam speed for the data collection, a slow scanning speed was preferred in order to reduce the noise at each measurement point during a single scan. The use of a small aperture and a large working distance was also important to ensure the maximal depth of field over the whole surface of the welded wires.

It should be noted that two-dimensional (2-D) image correlation techniques have been used to investigate the deformation of a non-plane surface that undergoes some out-of-plane motion, such as a rotation of the sample around its axis. However, such motions have little influence on the evaluation of the axial strains of the sample that is focused on, as the axis of the sample remains essentially parallel to the image plane. Transverse strains are more difficult to investigate because of the possible rotations of the sample around its axis, and so are not considered in this study.

\subsection{Nanoindentation}

Young's modulus and hardness were measured on a longitudinal polished surface with a Berkovich-type pyramidal indenter, using the continuous stiffness method detailed by Oliver and Pharr [13]. The maximal penetration depth of the indenter was set to $1 \mu \mathrm{m}$, with a penetration speed of $5 \mathrm{~nm} \mathrm{~s}^{-1}$. A harmonic amplitude of $2 \mathrm{~nm}$ was superimposed on this penetration motion in order to provide a continuous calculation of the Young's modulus and hardness over the experiment. At a small penetration depth, the measurements are quite noisy, as the area of contact between the indenter and the specimen section is very small. This noise decreases as the indenter penetrates deeper into the material and the tested volume becomes more representative of the polycrystalline specimen. For these reasons, the values given in the results section were averaged over the last stage of the inden- tation curve. A rectangular region around the weld was mapped with a regular grid of indentation points in order to reveal inhomogeneities of the mechanical properties of the weld and of the base wires.

\subsection{Modelling}

The in situ tensile test observations were compared with mechanical numerical simulations performed with the Abaqus $^{\odot}$ software. The simulated domain was axisymmetric and included the weld area $(250 \mu \mathrm{m})$ and two $100 \mu \mathrm{m}$ long regions on both sides in the SS and NiTi wires. The mechanical properties of each region were obtained from the nanoindentation measurements in the weld and base wires. The external cylindrical surface of the domain was free to move (no stress), while one cross-section of the wire was fixed and the other one was prescribed to move at a constant rate.

\subsection{Automated digital image correlation}

Finally, as a validation to the strain computation, 2-D SEM SE images of the specimen surface at various strain levels were analysed using automated DIC. These calculations were made using CMV, a specific software developed at ENPC [14].

The images were subdivided into square juxtaposed correlation windows. Their size, $30 \times 30$ pixels, was chosen such that each window contained enough natural image contrast for automated pattern matching. This contrast was essentially due to surface imperfections induced by grinding (asperities, inclusions, scratches, etc.). The wire was discretized into 30 windows along its diameter. The motion of each window was deduced by standard DIC algorithms, making use of an affine shape function and bilinear grey level interpolations to reach subpixel accuracy in the displacement evaluations [14]. Once the displacement of the centre of each window was determined, the strain field was computed using an 8-neighbours scheme in order to reduce the resulting standard deviations, following the principles described in Allais et al. [15]. Because the natural contrast of the images was low, the resulting accuracy was below that which is usually expected from DIC algorithms, but was nevertheless sufficient to quantify and compare strains in various parts of the wire. Note that the mesh was interrupted along the crack as the deformation deduced from the crack opening is singular. Thus, the measurements near the crack are less accurate than the others.

\section{Results}

The experimental results presented in this section follow the same order as that adopted in the previous section.

\subsection{In situ tensile testing}

Preliminary tensile tests using a conventional machine revealed an unusual location of the fracture [12]. Indeed, 
in all the samples tested, a crack appeared at the beginning of the superelastic plateau, in a region near the surface, between the weld and the NiTi base wire. It then propagated along a circle over the whole perimeter of the weld, while the centre of the welded wires remained linked. Based on these observations, several hypotheses could be envisaged: (i) the crack initiates at some surface defect, e.g. impurities, defects associated with pulse overlaps, some small roughness due to solidification shrinkage and liquid surface oscillations; (ii) stress concentration occurs in this region since the weld section is slightly reduced compared to the nominal section of the base wires; (iii) deformation is substantially increased in this region due to the inhomogeneities of the mechanical properties of the base wires and weld region. In order to eliminate the first two conjectures, the section of the welded samples was first reduced to $200 \mu \mathrm{m}$ by mechanical grinding before in situ tensile testing by SEM.

During a classical tensile test in a scanning electron microscope, the stress-strain curve shows a standard superelastic behaviour (see Fig. 3a), with three distinct zones: an initial elastic domain, a superelastic plateau and finally a very short increase in stress at the end of the plateau before complete rupture of the specimen.

The elastic domain is linear and reversible, with an effective Young's modulus $\langle E\rangle$ corresponding to two wires put in series, if one neglects the small welded area itself. If the lengths of NiTi and SS in between the grip are the same, one has $\langle E\rangle^{-1}=0.5\left(E_{1}^{-1}+E_{2}^{-1}\right)$, where $E_{1}$ and $E_{2}$ are the Young's modulus of the two base wires. No noticeable damage could be observed until the superelastic plateau is reached (see Fig. 3b, and the corresponding position on the stress-strain plot).

Right at the onset of the superelastic plateau (see Fig. 3c), a crack initiates at the surface of the specimen, in the transition zone between the NiTi wire and the weld region (see Fig. 3c). Then the crack does not evolve until the end of the plateau and the only visible change is the reduction in the NiTi diameter, since the reversible martensitic transformation occurs at a constant volume [16].

Finally, at the end of the superelastic plateau, when the stress in the specimen starts increasing again, the crack propagates over the whole perimeter of the specimen along a circle, still in the region of transition between the weld and the NiTi wire. This crack opening instantaneously leads to a reduced section of the specimen and thus an increased stress. After a small displacement increment, the central part of the specimen also breaks. The fracture surface corresponds roughly to a cross-section of the specimen; therefore the final failure occurs in the NiTi and a small portion of NiTi remains attached to the weld area (see Fig. 2 for the shape of the weld).

The sample shown in Fig. 4 was deformed until the end of the superelastic plateau (stage 3 in Fig. 3). After polishing a longitudinal section, the crack that propagated over the entire perimeter of the specimen appears on both external sides of the section (dashed white circles). The crack ini-

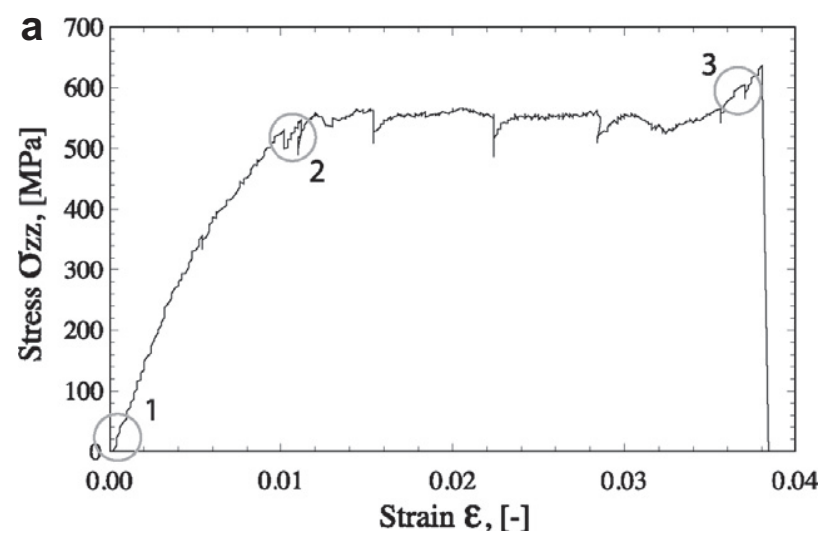

b

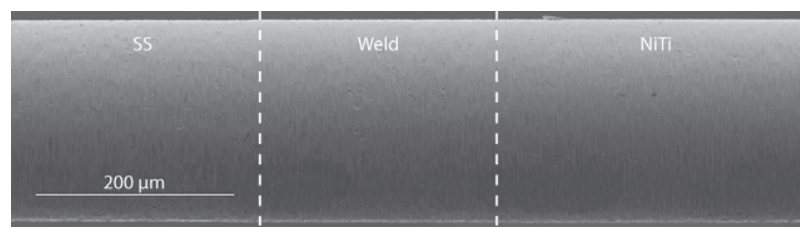

C

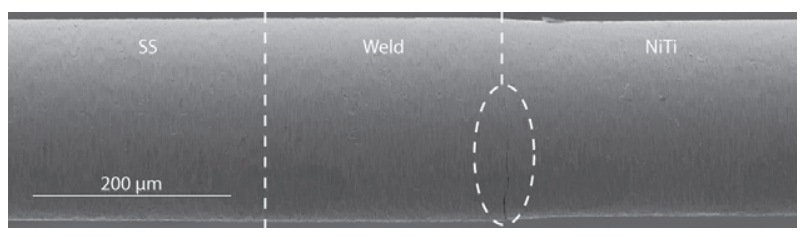

d

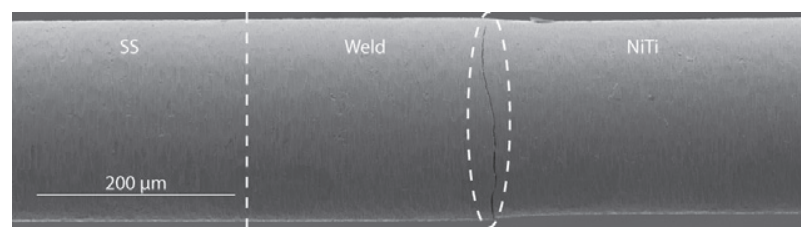

Fig. 3. In situ tensile testing data and SEM SE micrographs at critical stages of deformation. (a) Typical tensile test of laser-welded NiTi-SS $300 \mu \mathrm{m}$ wires, then reduced to $200 \mu \mathrm{m}$ by mechanical grinding. The circles numbered 1-3 correspond to the following deformation stages. (b) Stage 1: SE image of the initial state after $10 \mathrm{~N}$ preloading in order to ensure good grip positioning. (c) Stage 2: SE image at the beginning of the superelastic plateau: the crack initiates at the first kink in the circled area. (d) Stage 3: SE image at the end of the superelastic plateau, just before complete fracture of the specimen : the crack has propagated over the whole perimeter of the specimen.

tiates in the weld near, but not exactly at, the interface with $\mathrm{NiTi}$, and propagates nearly parallel to the weld-NiTi interface until it reaches the NiTi base wire.

The broken surfaces of several specimens were reconstructed in three dimensions using the $\mathrm{Mex}^{\circledR}$ software and three SE images taken at various projection angles $\left(-7^{\circ}, 0^{\circ}, 7^{\circ}\right)$. Fig. 5 reveals the crack propagation path after initiation in the outer ring of the weld, viewed from the NiTi wire side. As the crack is first initiated at the transition between the welded zone and the NiTi wire, this side of the specimen shows the crack in the weld and in the central part of the NiTi wire. The outer ring has the average composition of the weld (assessed by EDX analysis), and exhibits a brittle rupture visible by the succession of flat surfaces with steps in between. The central part is made 


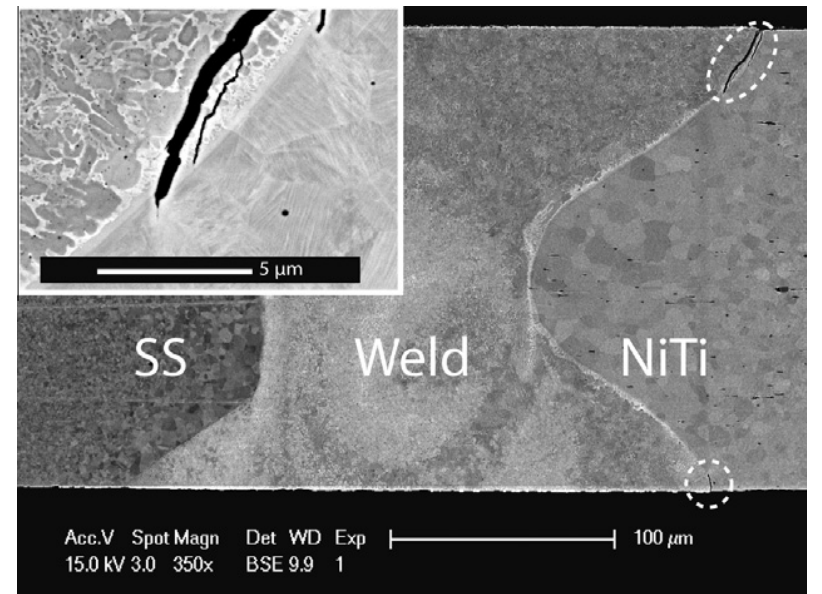

Fig. 4. SEM BSE image showing a longitudinal cut of a welded NiTi-SS couple deformed up to the end of the superelastic plateau. The two dashed white circles reveal that the crack has propagated over the whole perimeter of the specimen and the enlarged view shows the tip of the upper part of the crack.

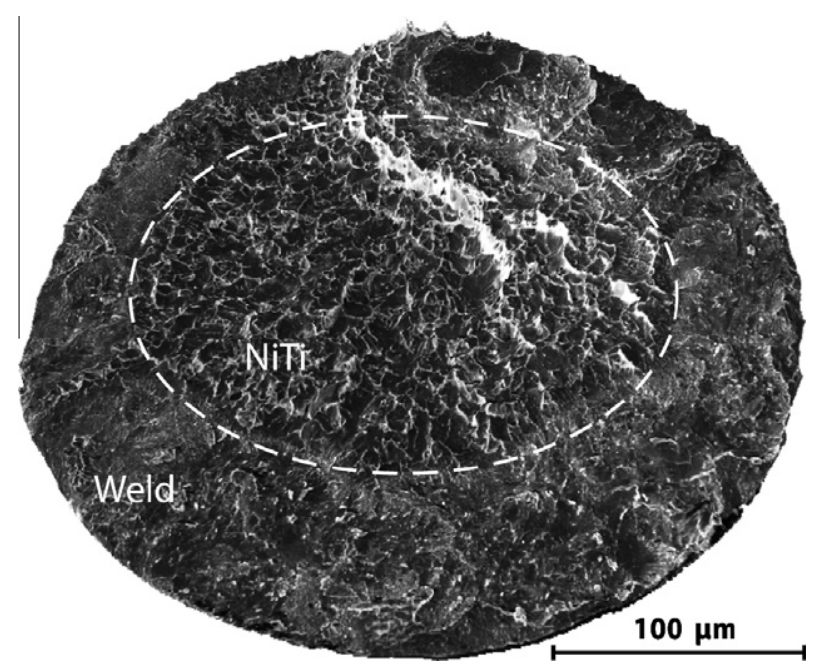

Fig. 5. Three-dimensional reconstructed view of the broken surface viewed from the NiTi wire side.

only of NiTi, thus proving that the X-shape of the weld shown in Fig. 2 is strong enough to sustain the stress and that the final rupture occurs in the NiTi wire. The surface of the NiTi still attached to the weld shows a typical ductile fracture.

\subsection{Nanoindentation}

To gain a better understanding of the internal stresses developing in the specimen during deformation, mechanical computations were performed with Abaqus ${ }^{\odot}$ using the inhomogeneous properties measured by nanoindentation. Three domains were considered: the SS region, the NiTi region and the welded zone itself, which was assumed to be homogeneous in composition. This assumption was validated by EDX measurements, which revealed no major
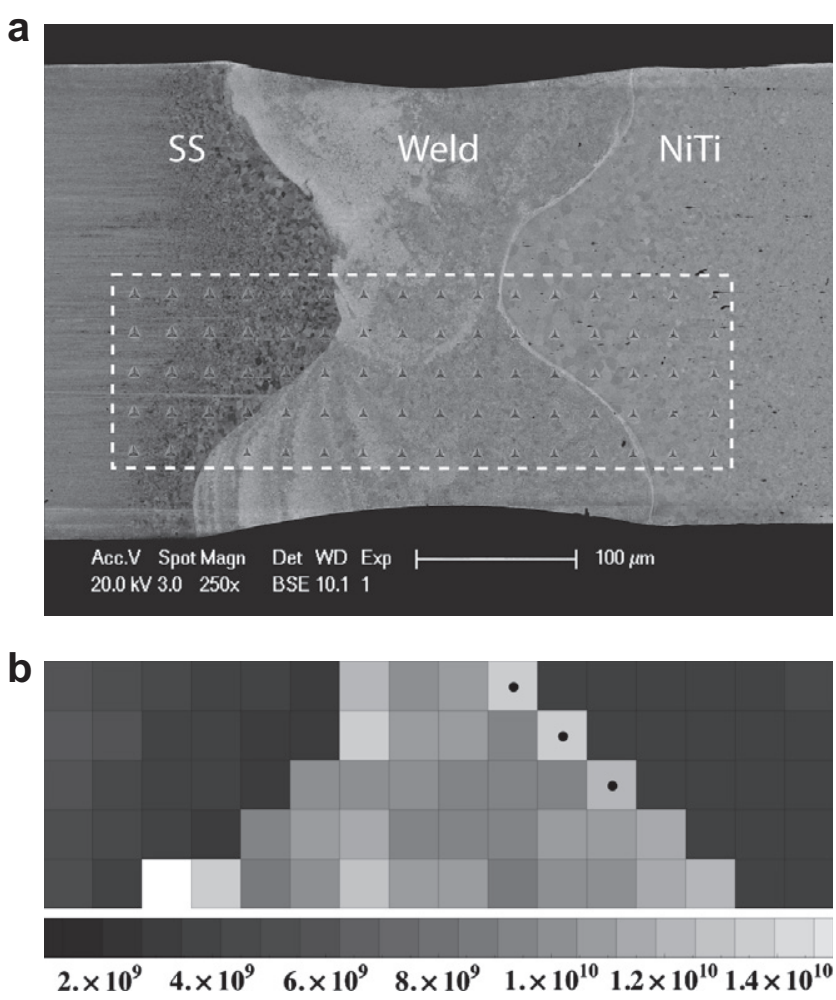

C

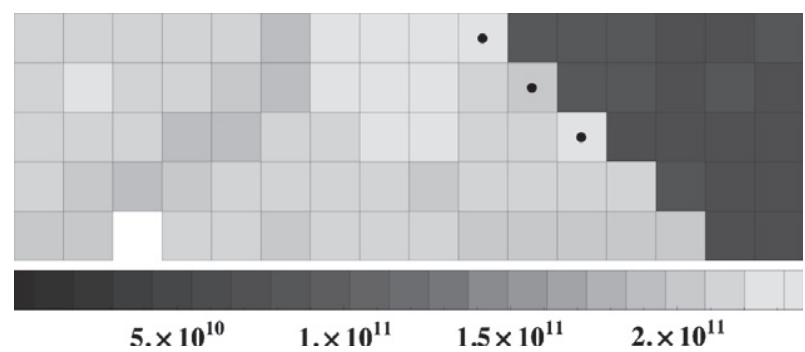

Fig. 6. Indentation test on a longitudinal section of a NiTi-SS laser weld. (a) BSE image of a longitudinal section which was indented in the dashed white rectangle with a square grid spacing of $25 \mu \mathrm{m}$. (b) Hardness plot $\mathrm{H}$ (Pa) deduced from the indentation test in the white rectangle shown in (a). (c) Young's modulus plot E deduced from the indentation test in the white rectangle shown in (a).

composition variations over the welded zone, except for the small white areas, which have a higher chromium content (see Fig. 7).

Assuming axisymmetric properties, the zone outlined by the white rectangle of the longitudinal section shown in Fig. 6 was mapped with five rows of 16 indentation points. The indentation points were made on a square grid of $25 \mu \mathrm{m}$ spacing (see Fig. 6a). The third position of the bottom row was not indented because of an instrumental problem during the measurement. The local hardness $H$ of the material is not required for the mechanical computations, but it was nevertheless measured and used to deduce the corresponding yield stress $\left(\sigma_{y}\right)$ according to the approximate relationship:

$\sigma_{y} \approx \frac{1}{3} H$ 
Table 1

Mean Young's modulus $(E)$, yield stress $\left(\sigma_{y}\right)$ and total number of indentations $(N)$ for the three modelling domains (NiTi, weld, SS), as obtained from nanoindentation experiments, with their respective standard deviations.

\begin{tabular}{lrll}
\hline & $E(\mathrm{GPa})$ & $\sigma_{y}(\mathrm{GPa})$ & $N$ \\
\hline NiTi & $75 \pm 4$ & $1.24 \pm 0.05$ & 20 \\
Weld & $216 \pm 6$ & $3.62 \pm 0.36$ & 36 \\
SS & $208 \pm 8$ & $1.38 \pm 0.29$ & 23 \\
\hline
\end{tabular}

The average Young's modulus $E$ and yield strength $\sigma_{y}$ of the three zones, i.e., SS, NiTi and the weld region, are summarised in Table 1. As can be seen in the maps of Fig. 6, the SS base wire shows a slightly reduced hardness (or yield strength) near the weld. This corresponds to the heat affected zone (HAZ), since the high $\sigma_{y}$ of the base SS is mainly due to the cold work during the wire drawing. During laser heating, recrystallization occurs and reduces the yield strength. The grain size increase due to heating is clearly visible in the SEM micrograph of Fig. 4, especially near the centre of the SS wire. The Young's modulus does not show such an effect, since it is a more intrinsic property of the material. The hardness of the NiTi wire is not affected in the HAZ, since the wires were annealed after drawing in order to recover their superelastic properties.

Inside the welded area, small variations of the hardness and Young's modulus can be seen, especially an increase in these properties in the area where the SS content is high (brighter parts of the weld seen in BSE due to higher $\mathrm{Cr}$ and $\mathrm{Fe}$ compositions). These variations are nevertheless small $( \pm 10 \%)$ compared with the large increase between the weld hardness and that of the base wires (see Table 1). For these reasons, only the average values listed in Table 1 will be used in the mechanical calculations.

It is interesting to link these mechanical properties with the local composition measured by EDX (see Fig. 7a). Compositions were subdivided into SS- and NiTi-rich areas, the former having a higher elastic modulus than the latter. At the weld-NiTi interface (on the right of Fig. 7a), a hardness increase can be observed (see the points outlining the three light grey squares with $H \geqslant 12 \mathrm{GPa}$, in Fig. 6b). At this interface, a thin white layer is visible at the microscopic scale in Fig. 6a, with the phase composition and density giving a brighter contrast than the average NiTi (see Fig. 7).

\subsection{Modelling}

For the stress-strain calculations performed with Abaqus $^{\circledR}$, the welded specimen was assumed to be axisymmetric, although the welding process itself is not since it was made by a succession of spot welds during rotation of the wire couple. This is a reasonable assumption since the process is in a quasi-steady state (i.e. nearly constant base temperature of the wires) after a few spot welds have been made, and any reheating of a spot weld by the next ones
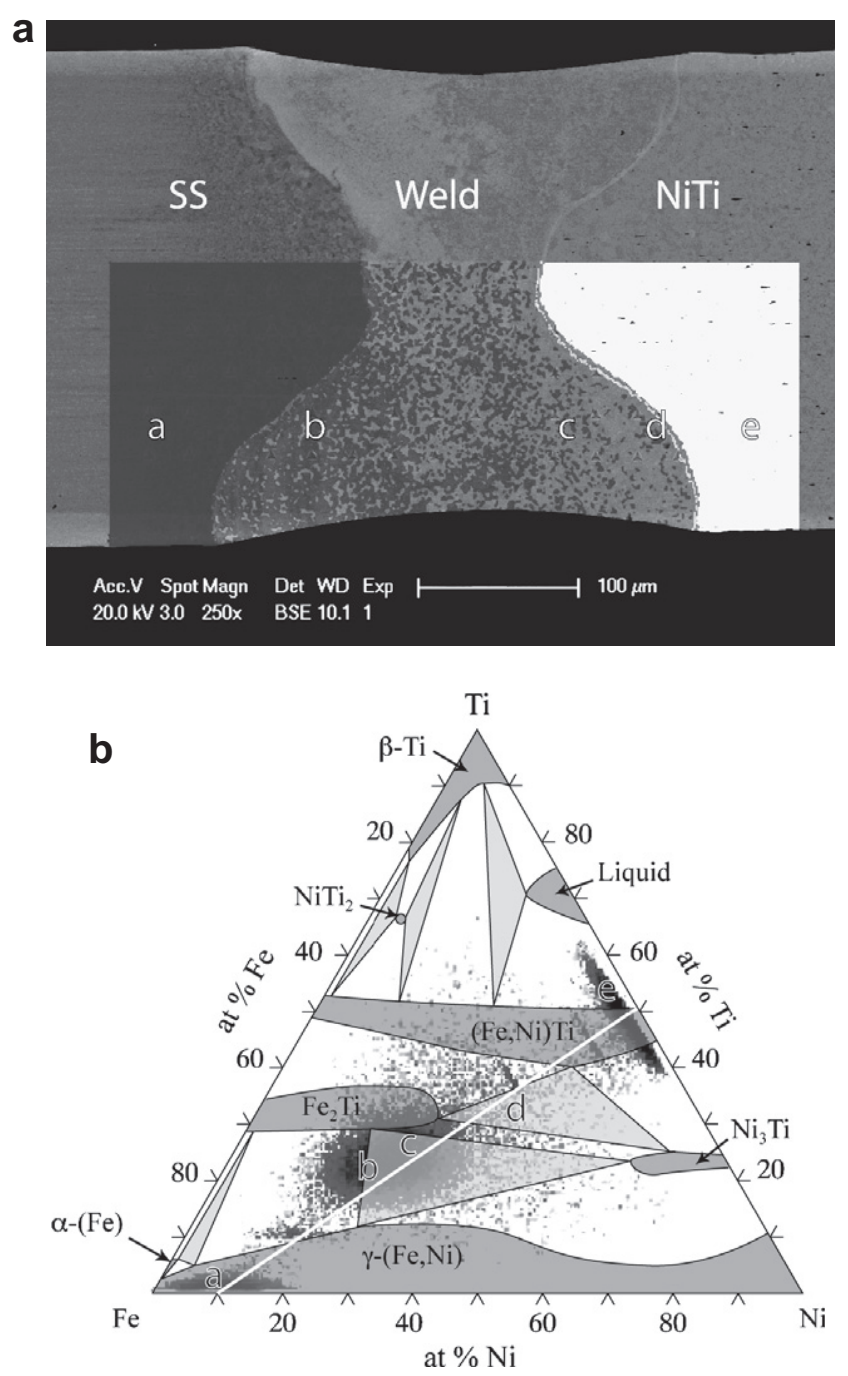

Fig. 7. EDX chemical analysis of the region near an SS-NiTi weld put into relation with the corresponding $\mathrm{Fe}-\mathrm{Ni}$-Ti ternary phase diagram. (a) Chemical EDX analysis of a typical weld area superimposed on the SE image. The grey levels indicate the local compositions, the dark region corresponding to SS and the white one to NiTi. These regions are also identified with the letters (a)-(e), and are reported in the Fe-Ni-Ti Gibbs simplex of (b). (b) Gibbs simplex of $\mathrm{Fe}-\mathrm{Ni}-\mathrm{Ti}$ similar to Fig. 1 with superimposition of the chemical compositions measured by EDX in zones (a)-(e) of the weld area.

has a minor influence compared to the mixing of the two base materials. Half a longitudinal section was therefore enmeshed over the length of the welded zone $(250 \mu \mathrm{m})$ and $100 \mu \mathrm{m}$ on each side of the weld. Three materials (NiTi, weld, SS) were considered, with their properties determined by nanoindentation (see Section 3.2). NiTi was modelled as an elastic medium up to superelastic stress (550 $\mathrm{MPa}$ ), followed by an ideally plastic regime up to $8 \%$ strain with a Poisson ratio $v=0.5$ since the austenite-martensite transformation occurs at constant volume [16]. After the superelastic plateau, the strain was modelled by an elastic domain. This elastic-ideally plastic-elastic approximation is only valid upon monotonic loading of the specimen since the superelastic plateau would be 
omitted during unloading. It was chosen because of the restrained possibility of Abaqus ${ }^{\odot}$ to manage superelasticity. Both the welded zone and the SS wire were treated as brittle elastic domains. As both domains have a higher Young's modulus than NiTi and a higher yield stress than the superelastic limit, most of the strain will be localized between the NiTi wire and the welded zone. The interface between the domains was approximated by a sinusoidal boundary fitted to the actual micrographs (see Fig. 7a).

Fig. 8 shows the radial component $\sigma_{r r}$ and the hoop component $\sigma_{\theta \theta}$ of the stress tensor calculated after $5 \%$ total deformation. As can be seen from the highly reduced diameter of the NiTi wire associated with a deformation at constant volume, the superelastic plateau of NiTi has been crossed. Although this deformation stage corresponds to point (3) of Fig. 3a, the 5\% overall deformation of the simulation cannot be directly compared to the $3.5 \%$ measured at the end of the experimental tensile curve, since the weld region is much more important in the $450 \mu \mathrm{m}$ simulation domain than in the $5 \mathrm{~cm}$ grip gap of the in situ experiment. Moreover, due to the contact with the much harder weld domain, the largest stresses are confined to this region. The significant difference in the deformation behaviour of these two neighbouring domains also explains the location

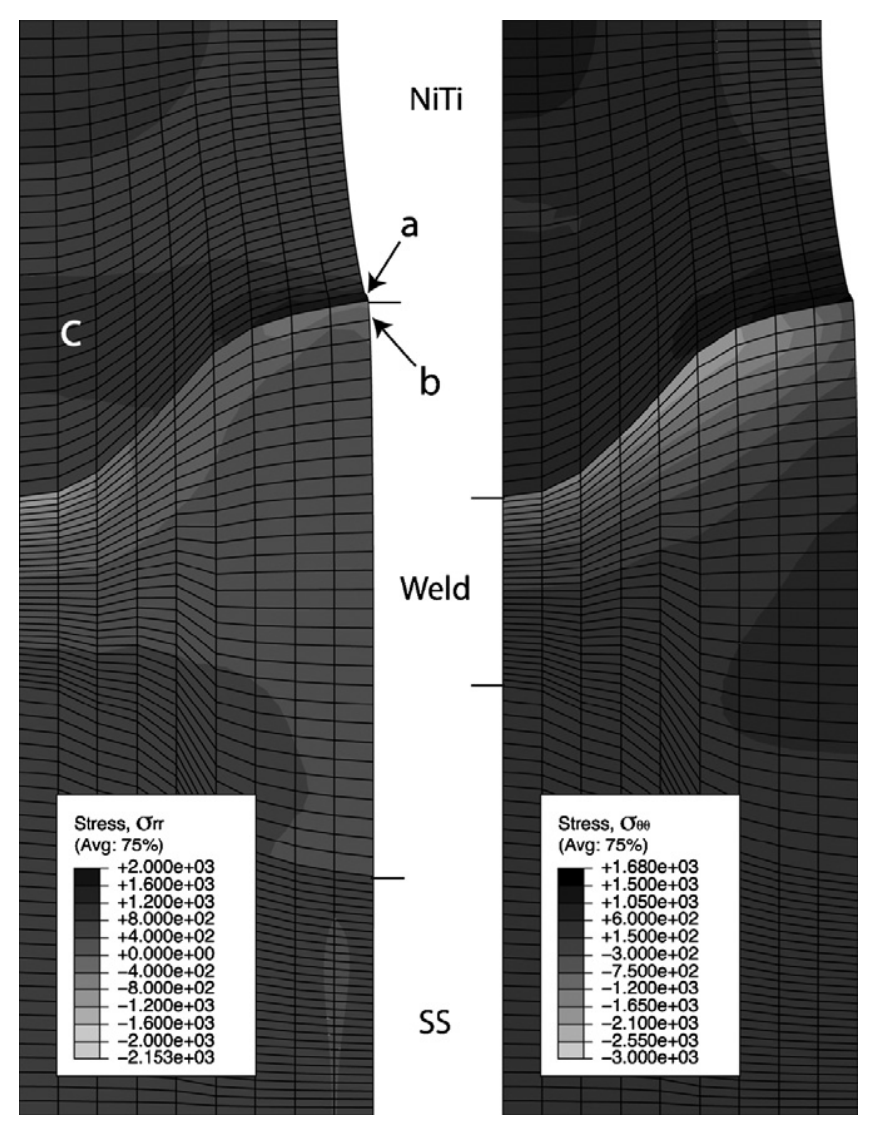

Fig. 8. Axisymmetric stress calculation of the welded zone, the NiTi wire on top and the SS wire at the bottom, showing the radial (left) and hoop (right) components of the stress tensor. The macroscopic average strain at this point is $5 \%$. of the crack initiation. Indeed, due to its deformation at constant volume once the superelastic plateau has been reached, the NiTi wire is in tension not only along the axial component of the applied load, but also in both the radial and hoop components (region labelled (a) in Fig. 8). Note that the outer ring of the weld region (region labelled (b)) has a compressive radial component. The axial tensile component of the stress tensor in the weld combined with the radial compression component initiates the crack in this zone. The computed axial stress $\sigma_{z z}$ showed a maximum near this location, which explains the preferential initiation site for the start of the austenite-martensite phase transformation.

In addition, the radial component of the stress tensor in the NiTi domain is fairly uniform near the centre of the wire, but exhibits a maximum in the region labelled (c), which is not closest to the weld. This somehow shields the stress in the tip region of NiTi closest to the weld near the centre of the wires and explains why the crack propagates through the NiTi wire near the centre of the welded specimen, rather than along the $\mathrm{NiTi}-$ weld interface (see Fig. 5).

\subsection{Automated digital image correlation}

Image correlations were used as a validation (and comparison) of the stress-strain calculations. Indeed, displacements of surface defects and singularities can be tracked between successive images, and from that components of the strain (and rotation) tensors can be deduced. It should be borne in mind that such measurements were made at the surface of the sample (see Fig. 3b-d) and not, of course, on longitudinal sections.

Fig. 9 shows the obtained strain field at position 3 of the tensile test described in Fig. 3a (see the corresponding SE image in Fig. 3d). The external contour of the welded wires and the final crack are also drawn (black lines) in order to clearly localize the position of the measured strains. The tensile component of the strain tensor, $\varepsilon_{z z}$, is displayed with squares of various grey levels (scale on the left), the centre of which is located on the deformed position of the initially equally spaced square grid. A bimodal strain repartition is clearly visible: the SS wire and weld region are deformed less than $1 \%$ (dark regions), while the NiTi wire experiences a deformation of $9-11 \%$ (light grey region). This corroborates the computed stress-strain state of Section 3.3, as the NiTi wire accommodates the deformation by crossing its superelastic plateau. Near the crack, the NiTi surface is relaxed and exhibits a lower axial strain (intermediate grey level region).

In order to better understand the macroscopic superelastic plateau observed in Fig. 3a, a more detailed analysis of the deformation measured by image correlation was performed between position (2) (beginning of plateau) and position (3) (end of the plateau). The NiTi wire deformation component $\varepsilon_{33}$ between these two images was averaged over a subdomain shown by the rectangle drawn on 


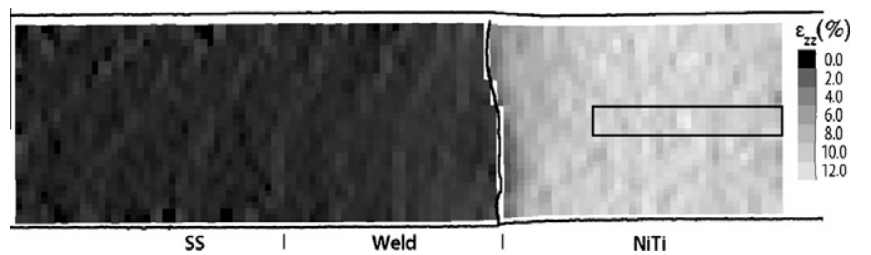

Fig. 9. Strain field measured by automated image correlation in a longitudinal section of a welded couple during in situ tensile testing, between the initial state (unloaded, Fig. 3a, position 1) and the final state (post-superelastic plateau, Fig. 3a, position 3). The longitudinal component of the strain tensor is shown with various grey level squares, each of them being centred on the deformed position of the initially equally spaced square grid.

the right of Fig. 9. Surprisingly, while the macroscopic deformation of the whole grip gap increases from $1 \%$ (position 2) to $3.6 \%$ (position 3) (see Fig. 3a), the average local deformation $\left\langle\varepsilon_{33}\right\rangle$ measured within the rectangle between these two states is only $0.2 \%$. This shows that the massive transformation had already occurred in this region, just before position (2) of the tensile curve, and that the macroscopic superelastic plateau measures the progression of this phase transformation along the whole length of the NiTi wire. The small deformation measured in the rectangle corresponds to the small deformation increase once the whole NiTi wire has transformed into martensite (see Fig. 3a).

\section{Synthesis}

From all these observations and measurements, a description of the fracture mechanism can be made based on a composite model whose details can be found in the Appendix. Since the SS-weld interface is not relevant to the crack and the Young's moduli of the corresponding phases are quite similar (see Table 1), this composite model only considers the NiTi wire, called medium 1 in the Appendix, and the (SS + weld) domain, called medium 2 . The sinusoidal shape of the interface between these two domains is approximated by a stair-like function of thickness $L_{3}$ (see Fig. 10). This simple model thus considers two media, 1 and 2, put in series, with a composite in between made of media 1 and 2 in parallel with respect to the tensile axis. Medium 1 has a Young's modulus about one-third of that of medium 2. Once medium 1 enters the superelastic plateau, its stress is constant.

The medium 1 in the composite region of thickness $L_{3}$ is weaker than medium 2, most of the axial stress being carried by the latter. Since the NiTi (domain 1) facing domain 3 (stair-like interface domain) near the surface of the wire must have the same stress, it is the first region to deform superelastically at constant volume. Therefore, it tends to contract along the radial and circumferential directions, as shown in the simulations, thus adding some shear component at the periphery of the (SS + weld) domain. Considering the real sinusoidal shape of the interface, it is therefore not surprising that the maximum stress is in the

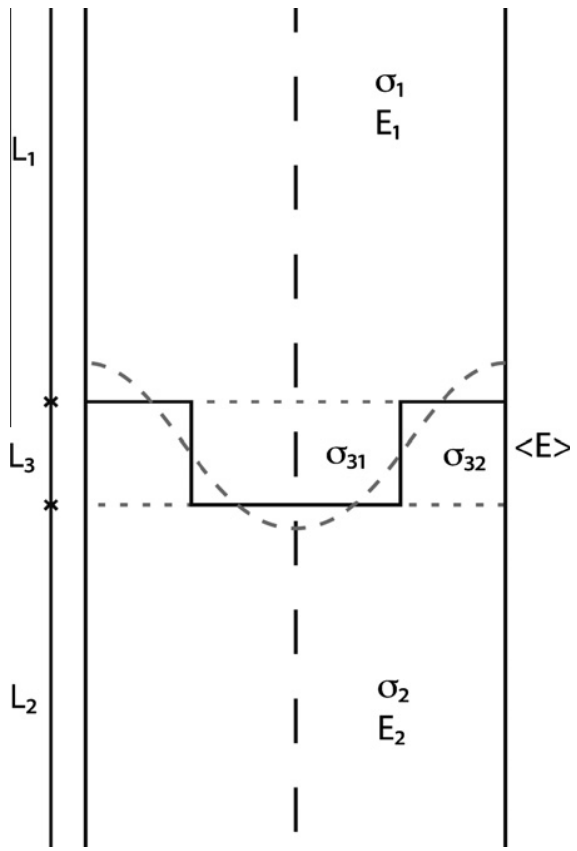

Fig. 10. Composite model used for a simple stress distribution analysis between NiTi (domain 1) and (SS + weld) (domain 2).

weld region at the outer surface of the wire, and consequently crack initiation occurs at this location.

\section{Conclusion}

In this research, the particular behaviour of cracks, which nucleate and propagate in dissimilar welds, was observed in NiTi-SS laser-welded submillimetric wire couples and analysed by SEM in situ tensile testing. Further chemical and mechanical investigations were conducted to identify the phases in the weld, and their intrinsic mechanical properties were explored by nanoindentation.

Computational models were designed to investigate the internal stress-strain states at critical points, such as the fracture location between the weld and NiTi base wire domains. The modelling showed a protective effect on the NiTi wire end near the weld, made by the bowl-shaped interface of the molten zone. Indeed, as the weld is mechanically stronger, its outer circumference sustains the radial stresses and thus protects the inner NiTi wire.

Finally, the sensitivity to localized failure is caused neither by the eventually weak weld properties nor by the section striction observed on the welded couple, but by the important property change at the interface between the weld and the NiTi base, and the complex shape of the latter.

\section{Acknowledgement}

This research was sponsored by the Swiss Confederation's Innovation Promotion Agency CTI (Project No. 8545.1). 


\section{Appendix A. Composite model}

In order to better understand the stress localization at the crack initiation point in the welded area, a simple composite model is developed here. For this purpose, only the interface between the NiTi and the weld is considered, the latter having the properties of SS (see Table 1), while its sinusoidal shape is approximated by a staircase (see Fig. 10). Domain (1) is the NiTi wire, while domain (2) corresponds to the (weld $+\mathrm{SS}$ ) region. From this simple geometric arrangement, an accommodation domain made of domains (1) and (2) in parallel can be identified (in between the dashed lines): it has a length $L_{3}$, corresponding roughly to the extent of the sinusoidal interface shape between NiTi and the weld region. Its average elastic modulus is labelled $\langle E\rangle$. The model considers the uniaxial component of the stress and strain only, and therefore a constant cross-section $S$ of the sample.

The equilibrium of forces along the axial direction imposes that:

$\sigma_{1}=\sigma_{2}=\left\langle\sigma_{3}\right\rangle=\sigma$

The stress $\left\langle\sigma_{3}\right\rangle$ in domain (3) is an average of the two phases, which can be deduced from the assumption that both phases in this region have the same strain:

$\left\langle\sigma_{3}\right\rangle=\left(\sigma_{31} \cdot g_{1}+\sigma_{32} \cdot g_{2}\right)$

where $g_{1}$ and $g_{2}$ are the corresponding surface fractions of phases (1) and (2) in domain (3), i.e. $g_{1}=S_{1} / S$ and $g_{2}=S_{2} /$ $S$ (see Fig. 10).

Using Hooke's law for the parallel configuration of the accommodation zone (3), this can be written also as:

$\langle E\rangle=E_{1} \cdot g_{1}+E_{2} \cdot\left(1-g_{1}\right)$

Since $\sigma_{32}=E_{2} \cdot \Delta L_{3} / L_{3}$, the stress intensity factor in the ( $\mathrm{SS}+$ weld) region in the accommodation domain (3) is given by:

$$
\frac{\sigma_{32}}{\sigma}=\frac{E_{2}}{g_{1} \cdot E_{1}+\left(1-g_{1}\right) \cdot E_{2}}
$$

With $E_{2} \cong 3 E_{1}$ (see Table 1 ) and $g_{1} \cong 2 / 3$, the stress intensity factor in the (SS + weld) region at the outer surface of the wire is about $9 / 5$, i.e. the stress in the region where the crack appears is nearly twice the applied stress.

\section{References}

[1] Duerig T, Pelton A, Stöckel D. Mater Sci Eng A 1999;273275:149-60.

[2] Hall PC. Method of welding titanium and titanium based alloys to ferrous metals. US 2004/0182835 A1; 2004.

[3] Pelton A, Russell S, DiCello J. JOM 2003;55:33-7.

[4] Cacciamani G, De Keyzer J, Ferro R, Klotz U, Lacaze J, Wollants P. Intermetallics 2006;14:1312-25.

[5] Kirkaldy JS, Brown LC. Can Metall Quart 1963;2:89-117.

[6] Uenishi K, Seki M, Kunimasa T, Takatsugu M, Kobayashi K, Ikeda T, et al. In: Proceedings of SPIE - the international society for optical engineering, vol. 4830; 2002. p. 57-62.

[7] Gugel H, Schuermann A, Theisen W. Mater Sci Eng A 2008;481482:668-71.

[8] Favez D, Wagnière J-D, Rappaz M. In: ICALEO 2008 - 27th international congress on applications of lasers and electro-optics, Temecula, CA; 2008. p. 327-31.

[9] Lee KJ, Kumai S. Mater Trans 2006;47:1178-85.

[10] Borrisutthekul R, Yachi T, Miyashita Y, Mutoh Y. Mater Sci Eng A 2007;467(1-2):108-13.

[11] Favez D. Ph.D. thesis, Ecole Polytechnique Fédérale de Lausanne; 2009.

[12] Vannod J. Ph.D. thesis No. 5120, EPFL; 2011.

[13] Oliver W, Pharr G. J Mater Res 1992;7:1564-80.

[14] Bornert M, Valès F, Gharbi H, Nguyen Minh D. Strain 2010;46:33-46.

[15] Allais L, Bornert M, Bretheau T, Caldemaison D. Acta Metallurgica Et Materialia 1994;42:3865-80.

[16] Otsuka K, Ren X. Prog Mater Sci 2005;50:511-678. 